\title{
An RFID Based Access Control System operating with a Computer Program and a Database
}

\author{
Waly Fall \\ Department of Physics \\ Faculty of Science and Technology, \\ Cheikh Anta Diop University of Dakar. \\ Dakar, Senegal \\ waly.fall@ucad.edu.sn
}

\author{
Djamal Abdoul Nasser Seck \\ Department of Mathematics and \\ Computer Science \\ Faculty of Science and Technology, \\ Cheikh Anta Diop University of Dakar. \\ Dakar, Senegal \\ djamal.seck@ucad.edu.sn
}

\author{
Fabé Idrissa Barro \\ Department of Physics \\ Faculty of Science and Technology, \\ Cheikh Anta Diop University of Dakar. \\ Dakar, Senegal \\ fabe.barro@ucad.edu.sn
}

Received: July 13, 2021. Received: December 3, 2021. Accepted: December 19, 2021. Published: December $27,2021$.

\begin{abstract}
This article focuses on the realization of an access control system based on RFID (Radio Frequency by Identification) technology. It is an authentication system for controlling access to a sensitive place. This system is composed of RFID cards which are badges that act as electronic keys, an RFID reader, an electronic lock, a microcontroller, a wifi module and a database installed on a computer. The identification number of an RFID card detected by the RFID reader is transferred, via the serial interface, to the microcontroller that communicates with the computer through the wifi module for verification in the database. If the information is valid, the microcontroller triggers the opening of the electronic lock.
\end{abstract}

Keywords-RFID, System, access control, identification, badge, reader, electronic lock, microcontroller, program, database

\section{INTRODUCTION}

The human being has always sought a reliable security system to protect himself and to protect his property. In the past, he used large stones and trees trunks to protect himself from dangerous animals and strangers. As techniques evolved, these rudimentary systems were replaced by doors and key locks. However, the mechanical key lock has security gaps, including the slow opening process, easy duplication of keys and lack of control. Today, the evolution of technology has allowed the development of more and more efficient security systems. An example of an application is access control to a sensitive area [1]. In this context, this paper will focus on the realization of an access control system based on RFID (Radio Frequency IDentification) technology [3] [8] to control the entry and exit of a sensitive area. After a study of physical access control techniques and RFID technology, we will talk about the realization of our RFID based access control system.

\section{PHYSICAL ACCESS CONTROL TECHNIQUES}

Physical access control allows the regulation of access to sensitive areas in order to protect people, property, information, etc. Physical access control systems [1] are generally based on an authentication mechanism through the use of code or password or through the use of biometrics (fingerprint recognition, optical iris recognition, voice recognition, facial recognition) or through the use of mechanical keys or access cards, etc. In this article, we focus on access control by RFID cards [7] which has some advantages: standardized technology, high security, accessible cost, ease of use, possibility of customization, durability, etc.

\section{STUDY OF RFID TECHNOLOGY}

Radio Frequency IDentification (RFID) [7] [10]. is a rapidly developing technology that uses radio waves for data collection and transfer.

\section{A. The components of an RFID system}

An RFID system consists of a tag (or RFID tag) and a reader. The tag, which is often integrated into a card or badge, consists of an electronic chip that stores an identification number and an antenna that ensures communication with the reader [3]. The tag is said to be passive if it has no internal power source. In this case it is powered by energy from the reader [3]. The tag is said to be active if it has an internal power source. In this case it is more autonomous and is able to have more energyconsuming functions [8]. As for the reader, it is also composed of an antenna to be able to exchange with the card and an integrated circuit that ensures the task of sending and receiving information.

Fig. 1 shows the components of an RFID system.

\section{B. How an RFID system works}

For the operation of the RFID System the RFID card and the reader communicate without physical contact by inductive coupling. In this communication the badge captures the electromagnetic waves emitted by the reader. Indeed, when a signal travels through an antenna, the current generates a magnetic field and therefore a magnetic flux. A receiving antenna placed near the transmitting antenna picks up some of this magnetic flux. It is said that the two antennas are coupled. However, the current flowing through the transmitting antenna varies over time, which implies the magnetic flux picked up by the receiving antenna also varies over time. This variation in flow creates an induced force that not only supports the feeding of the badge in passive communication but also translates the activity of the chip.

Fig. 2 illustrates how an RFID system works.

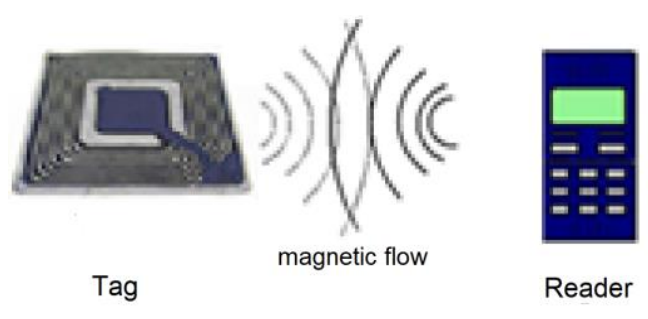

Fig. 1. The components of an RFID system 


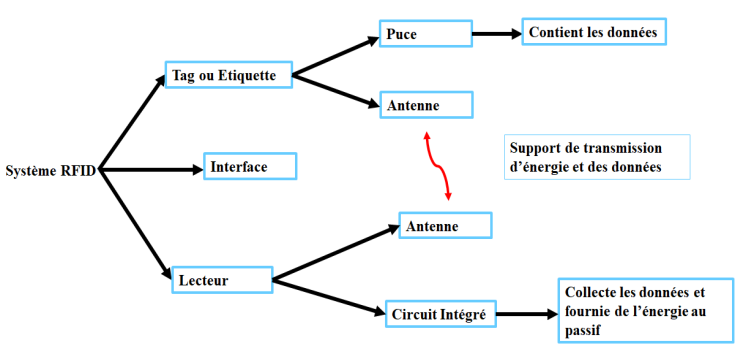

Fig. 2. How an RFID system works

Fig. 3 describes the electromagnetic phenomenon that exists between transmitting and receiving antennas. Some applications of RFID

RFID (Radio Frequency IDentification) was developed by the military in the late 1940s to identify aircraft and estimate their distance. Nowadays, it is still used in air traffic control in addition to other areas such as logistics [2] [4], healthcare [5] [6], retail or transport. Another interesting application is the location of animals or objects such as vehicles [9]. RFID has also developed rapidly in the field of security [1] [7]. For example, for better control of entrances and exits, access badges are used to replace traditional keys at the doors of residences, offices, etc. In this context, we propose a complete RFID-based access control system. The innovation in our system is that, in addition to an electronic part that we will detail later, it contains a computing part consisting essentially of a database that serves to store the information of the system for the control and identification of access cards and their users and to keep the access history. In this context, we propose a complete access control system consisting of an electronic part and a computing part.

\section{REALIZATION OF OUR ACCESS CONTROL SYSTEM}

In this section, we present our access control system based on RFID technology to control access to a sensitive area. The innovation in our system is that, in addition to an electronic part consisting of an RFID system, a microcontroller and other components that we will detail, it contains a computing part consisting essentially of a database that serves to store the information of the system for the control and identification of access cards and their users and to keep the access history.

\section{A. The Electronic components of our access control system}

The electronic part of our access control system is composed of badges that are RFID cards, an RFID reader, an electronic lock, a buzzer, a Led, a relay, a microcontroller.

Badges act as electronic keys. A badge is made from an ultra-white PVC offering a perfect resistance during repeated handling. It operates at $13.56 \mathrm{MHz}$. It is equipped with a chip and an antenna that allows data transmission with the RFID reader.

The model of the RFID Reader of our system is the MFRC522. This reader uses the $13.56 \mathrm{MHz}$ frequency band and its communication distance can be up to $6 \mathrm{~cm}$. It is powered by a low voltage of $3.3 \mathrm{~V}$ and current $13-25 \mathrm{~mA}$.

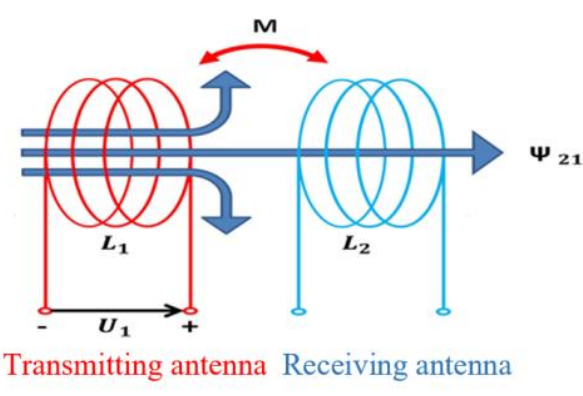

Fig. 3. Electromagnetic phenomenon between transmitting and receiving antennas

The electronic lock is a device that allows to lock or unlock the door. It is powered by an adaptor and the relay is used to cut or pass the power supply to the electronic lock.

. The microcontroller represents the brain of our system. It contains a microprocessor that performs the task of processing information and sending orders. It contains a memory in which runs the program that will operate the electronic lock. The model of the microcontroller of our system is the ESP32 which includes a wifi module for connection to the database. In terms of security the ESP32 microcontroller supports wifi encryption (WPA2). It is also equipped with a touch sensor, a temperature sensor and a Hall Effect sensor.

Fig. 4 shows the electronic components of the access control system.

\section{B. The database of the system}

The database of the system is implemented using the mysql DBMS and is installed on a computer connected to the system's microcontroller with a wifi connection. Fig. 5 shows the model of this database which consists of four tables: an administrator table that contains the attributes of the system administrators, a user table that contains the attributes of the people who may access with their badges to the sensitive area, a badge table that contains the attributes of all badges and an access table to keep the history of all accesses.

To access the sensitive area, a user must use a badge which is an RFID card with an identification number. An administrator can add one or more users to the database. He can delete or modify a user's characteristics. He can enable or disable a user's badge to allow or deny access to the sensitive area. The user presents his badge to the electronic lock which contains an RFID reader that will read the identification number. After checking in the database, if permission is granted, the date and time of access (entry or exit) are recorded. An administrator will then be able to view the access history to the sensitive area. 


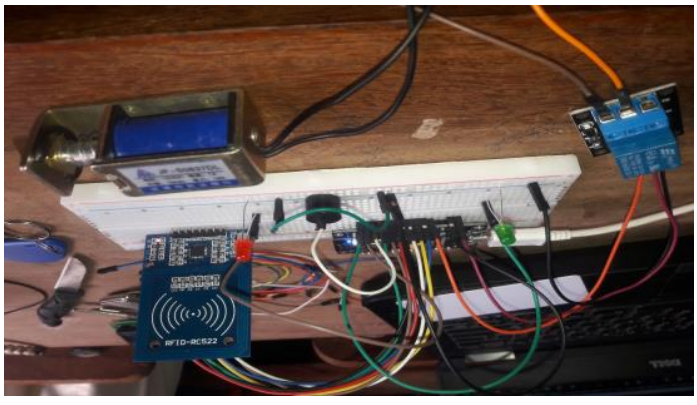

Fig. 4. Electronic components of the access control system

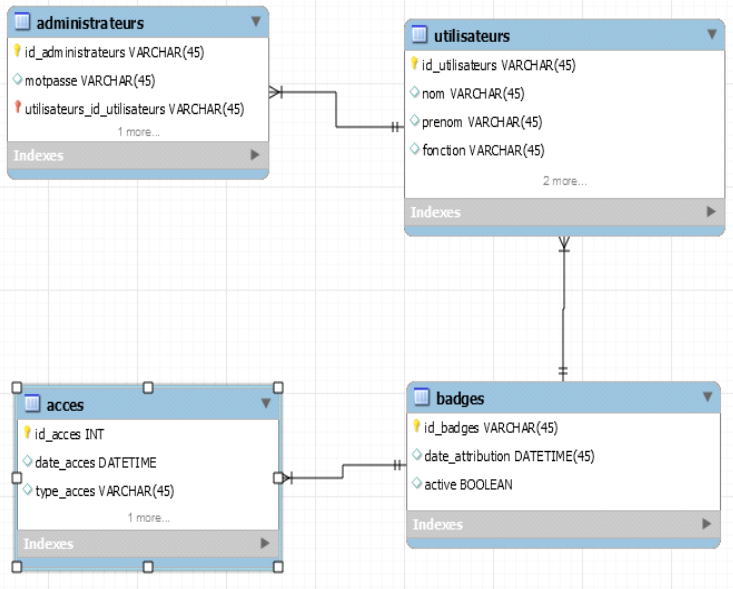

Fig. 5. The database model

\section{Architecture and operation of our RFID based access control system}

The components mentioned above are assembled in such a way that they form a complete access control system. The badges communicate with the RFID reader through electromagnetic waves at the frequency 13.56 Mhz. In addition, the reader is wired with the ESP32 microcontroller via the Serial Peripheral Interface (SPI) serial pins. The microcontroller contains a memory in which runs the program that will operate the electronic lock embedded in the door. The wifi module included in the microcontroller allows the transfer of information to the database. This assembly of the components constitutes the architecture of our access control system and is represented by Fig. 6 .

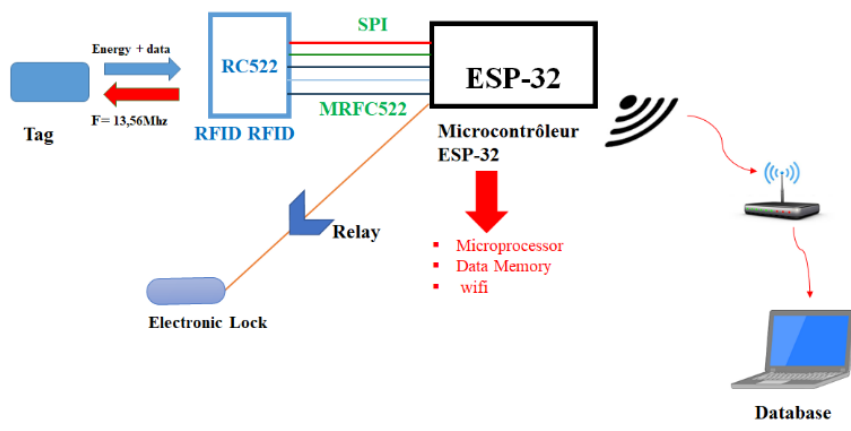

Fig. 6. Architecture of the RFID based access control system

\section{Operation of our access control system.}

Our access control system works through a $\mathrm{C}$ language program integrated into the microcontroller. The operation is described as follows.

As soon as a badge is detected by the reader, its identification number is transferred via the serial interface to the microcontroller and then to the database server via a wifi module for verification in the database. If access is allowed, a green LED lights up and the door opens. After the authorized person has access, the door closes automatically and the reader is waiting for another badge. If access is not allowed, the buzzer triggers a beep and a red LED lights up. In any case, the badge identification number is saved to keep track in the database. This functioning of the system is illustrated by the flow chart shown in Fig. 7 .

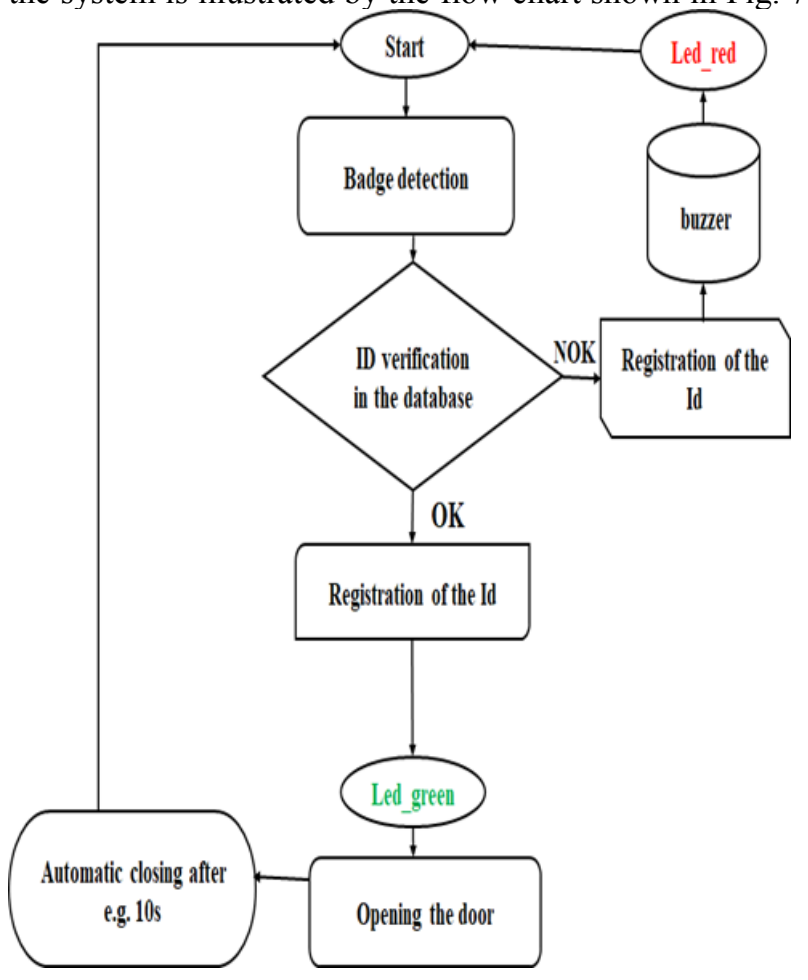

Fig. 7. Flow chart of the functioning of our access control system

\section{CONCLUSION}

In this article, we proposed an access control system based on RFID technology to control access to a sensitive area. This system is composed of badges that are RFID cards acting as electronic keys, an RFID reader, an electronic lock controlled by a microcontroller, a relay, a wifi module and a database installed on a computer. The database is used to store the system's information for the control and identification of access cards and their users, to keep access history, etc. This system allows the dematerialization of mechanical keys and locks that have known vulnerabilities.

As perspectives, it would be interesting to integrate into the system a passive infrared sensor (PIR) to detect movements in front of a door and a magnetic sensor to detect whether a door is open or closed. it would also be interesting to integrate a system of notifications of events by 
sms or by email. Deploying the database in the cloud would allow to monitor the system from any location with a web or mobile application.

\section{REFERENCES}

11] C. R. Aldawira, H. W. Putra, N. Hanafiah et al. Door security system for home monitoring based on ESp32. Procedia Computer Science, 2019, vol. 157, p. 673-682.

[2] I. Giusti, E. M. Cepolina, E. Cangialosi and al,. "Mitigation of human error consequences in general cargo handler logistics: Impact of RFID implementation", Computers \& Industrial Engineering, 2019, vol. 137, Article 106038.

[3] EPC Global Inc, Class 1 Generation 2 UHF Air Interface Protocol Standard Version 1.2.0", October 2008. [Online]. Available: http://www.epcglobalinc.org/standard

[4] M. Schmidt, L. Thoroe, and M. Schumann. RFID and barcode in manufacturing logistics: interface concept for concurrent operation. Information Systems Management, 2013, vol. 30, no 2, p. 100-115.

[5] [5] A. Abugabah, N. Nizamuddin, and A. Abuqabbeh. A review of challenges and barriers implementing RFID technology in the Healthcare sector. Procedia Computer Science, 2020, vol. 170, p. 1003-1010.
[6] [6] W. Yao, C-H. CHU and Z. LI. The use of RFID in healthcare: Benefits and barriers. In : 2010 IEEE International Conference on RFID-Technology and Applications. IEEE, 2010. P. 128-134.

[7] Y-Y. Chen, M-L. Tsai and J-K. Jan, "The design of RFID access control protocol using the strategy of indefinite-index and challengeresponse", Computer communications, 2011, vol. 34, no 3, p. 250256.

[8] M. Bolic, D.Simplot-Ryl and L. Stojmnovic, "RFID System : research trends annd challenges", John Wiley \& Sons, 2010.

[9] [9] Y. Ilam, T. Marie, P. Dondon. A Didactical Electronic Project For Graduated Students: Initiation To Autonomous Navigation Using A Small-Scale Model Elelectric Car. WSEAS Transactions on Advances in Engineering Education, vol. 16, 2019, pp. 28-35.

[10] V. P. Plessky and L. M. Reindl. Review on SAW RFID tags. IEEE Transactions on Ultrasonics, Ferroelectrics, and Frequency Control, vol. 57, no. 3, pp. 654-668, March 2010, doi: 10.1109/TUFFC.2010.1462.

\section{Creative Commons Attribution License 4.0 (Attribution 4.0 International, CC BY 4.0)}

This article is published under the terms of the Creative Commons Attribution License 4.0

https://creativecommons.org/licenses/by/4.0/deed.en US 